\section{Survival of the fittest or best adapted: HLA-dependent tumor development} Lisa Villabona,' Hildur Helgadottir,' Kjell Bergfeldt,' Federica Cavallo, ${ }^{2}$ Guido Forni, ${ }^{2}$ Soldano Ferrone, ${ }^{3}$ Aniruddha Choudhury,' Barbara Seliger, ${ }^{4}$ Rolf Kiessling'

'Dept. Oncology-Pathology, Karolinska Institute, Karolinska University Hospital, Stockholm, Sweden;

${ }^{2}$ Molecular Biotechnology Center, Dept of Clinical and Biological Sciences, University of Torino, Torino, Italy; ${ }^{3}$ University of Pittsburgh Cancer Institute, Departments of Surgery, Immunology and Pathology, School of Medicine, Pittsburgh, PA, USA;

${ }^{4}$ Institute of Medical Immunology, Martin Luther University Halle-Wittenberg, Halle/Saale, Germany

\section{Abstract}

The major histocompatibility complex (MHC) comprises a set of genes that are critical to immunity and surveillance against neoplastic transformation. There is increasing evidence that the $\mathrm{MHC}$ antigens not only regulate antitumor immune responses in experimental animal models but directly correlate with survival and prognosis of patients with diverse types of cancers. MHC antigens may in the future function as potential biomarkers for prognosis and allow selection of cancer patients for intensive therapy.

\section{Introduction}

The recognition of antigenic peptides by $\mathrm{CD}^{+} \mathrm{T}$ lymphocytes in the context of major histocompatibility complex (MHC) class I and class II molecules on the surface of antigenpresenting cells is one of the initial steps that prime the adaptive immune system to respond to invading pathogens as well as potentially cancerous cells. The MHC class I molecules are, therefore, essential components of the adaptive immune system ${ }^{1}$ and critical to the immunological recognition of tumor cells. As a result, defects in the expression and/or function of MHC antigens allow tumor cells to escape from recognition and destruction by the host's immune system. ${ }^{2-6}$

However, the number of studies analyzing associations between HLA frequency and cancer incidence, as well as prognosis, are still limited.

\section{The major histocompatibility complex}

In humans, the major histocompatibility complex is located on the short arm of chromosome 6 (6p21.3). It is one of the most intensively studied chromosomal regions in humans. Recently, the human genome has been depicted in block-like structures characterized by areas of high linkage disequilibrium (LD) creating so-called haplotype blocks, separated by regions of low $\mathrm{LD}$, hypothesized to correspond to recombination hot spots. ${ }^{7-10}$ This arrangement greatly facilitates the discovery of genes that predispose individuals to diseases through 'haplotype-tagging' singlenucleotide polymorphism, reducing the number of markers needed in the mapping process. ${ }^{11}$ Haplotype blocks are an algorithmic definition of a region in the human genome characterized by reduced haplotype diversity or strong LD, rather than a biological phenomenon. The HLA loci typically span $3.6 \mathrm{Mb}^{12}$ and extend to $7.6 \mathrm{Mb}$ with the telomeric repeats. The adjacent sequences within the superlocus contain several genes regulating immune function and demonstrate synteny to the mouse MHC. ${ }^{12,13}$ About $28 \%$ of the expressed transcripts from the genes within the superlocus are potentially implicated in the immune response. For this reason, it is not unexpected that this genetic region is linked to a large number of autoimmune and immune-mediated diseases. ${ }^{14}$ One characteristic of the MHC locus is the high degree of $\mathrm{LD}$ found between alleles and the high degree of polymorphism within the genes encoding peptide-presenting molecules (HLADPB1, -DQB1, -DQA1, -DRB1, -C, $-B$ and $-A$ ). "Ancestral haplotype" is a term used to describe conserved haplotypes that appear to be identical among individuals not known to be directly related. These ancestral haplotypes have a specific content of alleles at all MHC loci and have a particular genomic length. In the Caucasoid population, some 30 ancestral haplotypes and recombinants among them account for almost $90 \%$ of the haplotypes found during genetic family studies. ${ }^{15}$

\section{Association of MHC antigens with cancer prognosis}

The association of HLA and cancer is now being established. Interestingly, none of the HLA (class I and II) alleles have been demon-
Correspondence: Giuseppe V. Masucci, Dept. Oncology-Pathology, Karolinska Institute, Karolinska University Hospital, SE-171 76 Stockholm, Sweden

E-mail: giuseppe.masucci@ki.se

Key words: ccancer, HLA, ancestral haplotypes, DNA, mRNA.

Received for publication: 15 March 2010.

Revision received: 19 April 2010.

Accepted for publication: 23 April 2010.

This work is licensed under a Creative Commons Attribution 3.0 License (by-nc 3.0).

(C) Copyright G.V. Masucci et al., 2010

Licensee PAGEPress, Italy

Journal of Nucleic Acids Investigation 2010; 1:e9 doi:10.4081/jnai.2010.e9

strated to be associated with an increased incidence per se of any cancer. However, individual alleles are known to be overrepresented in certain cancers or correlate with survival or prognosis. The number of studies are still limited and predominantly describe HLA A* 2 associated virus-related malignancies, such as in situ cancer of the vulva, ${ }^{16}$ cervical cancer ${ }^{17}$ and nasopharyngeal cancer. ${ }^{18}$

\section{History of HLA and prognosis}

In a study of 176 early-onset breast cancer patients and 215 ethnicity and age-matched controls, the HLA class II genes HLA$D Q B^{*} 03032$ and $H L A-D R B 1^{*} 11$ were absent or expressed at significantly lower frequency in the breast cancer patients suggesting that these two alleles may be "protective". ${ }^{19}$ In contrast, expression of the $H L A-D R 17$ gene in patients with ulcerative colitis associated with a higher risk of patients developing colorectal cancer. In this study, 114 ulcerative colitis patients or ulcerative colitis patients who had developed colorectal cancer were compared for expression of various HLA class II alleles. While the HLA DR17 gene was noted in a higher frequency in the ulcerative colitis-colorectal cancer patients, HLA-DR7, -DR1 or -DQ5 alleles were noted at a significantly higher frequency in ulcerative colitis "control" cases that did not develop colorectal cancer. ${ }^{20}$ In another study, women carrying the HLA-DQB1*0301 allele exhibited an increased risk of developing cervical intraepithelial neoplasia when infected by HPV 16 and this allele was also overrepresented in cervical intraepithelial neoplasia grade III cases in comparison to cervical intraepithelial neoplasia grade II cases. ${ }^{21}$ The HLA class I allele $A^{*} 02$ was associated with a reduced risk and HLA-A*01 with an increased risk of developing EBV positive Hodgkin's lymphoma. $^{22}$ The HLA-A*02 allele is associated with poor prognosis in non-small cell lung can- 
cer patients ${ }^{23}$ ovarian and prostate cancer and malignant melanoma ${ }^{24-26}$ while HLA-A*11 and DR6 were associated with poor survival in patients with head and neck tumors. ${ }^{27}$ Finally, HLA has been studied as a factor that could predict the response to immunological treatments, such as vaccine or cytokine therapy, especially in melanoma, ${ }^{28-33}$ renal cell carcino$\mathrm{ma}^{3436}$ and chronic myelogenous leukemia. ${ }^{37}$

\section{HLA-A2 and ancestral haplotypes as prognostic factors}

We have been studying the correlation of ancestral haplotypes as a prognostic factor in several different malignancies. HLA-A2 is independently a negative clinical prognostic factor in patients with advanced ovarian cancer. ${ }^{24}$ Moreover, an overrepresentation of the HLA-A2 phenotype was observed in both ovarian and prostate cancer in Swedish patients compared to the normal population. The higher frequency of this allele found in Scandinavian countries significantly decreases moving further south in Europe. In parallel, ovarian and prostate cancer mortality rates decrease with latitude and correlate with the demographic changes in HLA-A2 expression. ${ }^{38}$

The relationship of HLA and cancer further extend the correlation of single alleles to cancer survival and prognosis. As described above, ancestral haplotypes represent clusters of alleles that occur at a higher frequency in certain populations than predicted. These ancestral haplotypes have been correlated with survival and prognosis in diverse malignancies. For example, the ancestral haplotype $8.1\left(\mathrm{~A}^{*} 01\right.$ B*08-TNF-308A) is predictive for a shorter progression free and overall survival in nonHodgkin's lymphoma ${ }^{39}$ or in malignant melanoma.$^{26}$ In another study performed by us, the ancestral HLA haplotype 62.1 [(A2) B15 Cw3 DRB1*04] in patients with advanced metastatic melanoma was studied. The effect of HLA types on prognosis defined by KaplanMeier and Cox-Mantel methods were analyzed in patients with stage III $(n=26)$ or IV $(n=65)$ melanoma: 62.1 ancestral haplotype in clinical stage IV patients was significantly and independently associated with the decreased survival rate from the time of appearance of metastasis $(\mathrm{HR}=2.14 ; \mathrm{CI}=1.02-4.4 ; \mathrm{P}=0.04)$ although the period from the primary diagnosis to metastasis was the longest in patients with this haplotype. Thus, melanoma patients in this cohort with the 62.1 ancestral haplotype which is associated with autoimmune diseases have an initial strong anti-tumor capacity with longer metastasis-free period but deteriorate rapidly when the disease advances. ${ }^{26}$

\section{The hypotheses}

The disease progression seems to be predetermined by a somatic gene such as HLA-A2.
At the genetic level one could consider a linkage to putative onco- or tumor suppressor genes. The HLA-locus at chromosome 6 is a region with high incidence of polymorphism and the classic illustration of linkage disequilibrium. ${ }^{40}$ One can thus imagine a genetic linkage to possible onco- or tumor suppressor genes present near the locus, i.e. CDKN1A p21/WAF1 on 6p21, TFA, AP2 on 6p24. A linkage to an onco- or tumor suppressor gene could explain the increased potential for oncogenic transformation of cells and thus an increased mortality in these patients.

At the epigenetic level a microRNA-dependent downregulation of the MHC antigenexpression in the HLA-A2 patients may be speculated. MicroRNAs (miRNAs) are an abundant class of small non-coding RNAs that function as gene regulators. Although deregulation of miRNA expression is involved in the initiation and progression of tumorigenesis, the underlying mechanisms of miRNA deregulation in human cancer are largely unknown. However Increasing evidence indicates that disturbance in the miRNA biogenesis machinery contributes to miRNA deregulation in human cancer. ${ }^{41}$ Reports of miRNA regulation of HLA-G expression have been recently published. ${ }^{42}$ In addition, a specific miRNA has been identified for HLA-A2 (miR181a) ${ }^{43}$ which incidentally is also reported to have tumor suppressor properties. ${ }^{44}$ During the initial process of oncogenesis, transformed cells may up-regulate transcription of the MHC genes which serve to absorb and engage the pool of miRNA. Potentially, this may prevent translation of MHC antigen message which impedes expression of MHC antigen and immune recognition. Moreover, normal regulatory functions of the miRNA are also compromised as the "decoy" transformed cells absorb and engage the available pool of miRNA. This sponge or decoy effect has been used as a method to study the function of miRNAs. ${ }^{45}$ On the other hand, it is still unknown whether this can occur naturally in a cell. Nevertheless, the above model could potentially explain the more progressing disease and poorer prognosis in HLA-A2 positive patients.

\section{Tumor escape, HLA expression and the antigen processing machinery}

\section{The escape}

Despite the attraction of T-cell based immunotherapies during the last decade, the clinical responses were effective only in a minority of immunized patients without a correlation between the induction of T-cell and clinical responses. ${ }^{46,47}$ These insufficient results may be due to tumor immune escape mechanisms. A number of distinct immune evasion strategies have been identified in tumors, which include defects in interferon signal transduction pathways, lack of co-stimulatory molecules and presence of co-inhibitory molecules, as well as HLA class I antigen processing machinery (APM) abnormalities, including haplotype loss. ${ }^{3,5,48,49}$ The impaired antigen processing and presentation lead to a reduced recognition of the HLA class $\mathrm{V} / \beta 2$ microglobulin $\left(\beta 2\right.$-m)-peptide complex by $\mathrm{CD} 8^{+}$ cytotoxic T lymphocytes ${ }^{50,51}$. Furthermore, in some malignancies the frequency of HLA class I APM downregulation is associated with higher grading, staging, aggressive histology and abnormal DNA content of tumors, which is often more pronounced in metastases when compared to primary lesions and associated with a reduced patients' survival. ${ }^{52-57}$ Matsushita et al..$^{58}$ found that a strong HLA-DR-positive expression on colorectal cancer cells was significantly related to better prognosis for the cancer patients. They have also suggested a possible role for c-myc and propose that HLA DR is up-regulated while c-myc is suppressed by interferon gamma (IFN- $\gamma$ ), thus decreasing levels of c-myc in cancer tissue with high levels of IFN- $\gamma$.

\section{Non-classical MHC}

HLA-E, - $F$ and $-G$ are non-classical MHC loci; the trans-membrane heavy chain class Ib and the association with the light chain $\beta 2$ microglobulin are their common characteristics.$^{59}$ HLA-G is physiologically involved in the regulation of fetal immunological escape from maternal immune surveillance and at low levels or in its absence is known to be associated with pre-eclampsia and spontaneous abortions. ${ }^{60,61}$ HLA-G is expressed in both membrane bound and soluble forms and has been described to function both as a tumour marker and prognostic factor. It is generally thought to provide the same immune escape mechanism to several human cancers. ${ }^{62,63}$ It may also be a promising target for immunotherapy. ${ }^{64}$ HLA-G binds directly to the inhibitory receptors ILT-2 on B, NK and T-cells, ILT-4 on macrophages, KIR2DL4 on NK cells and BY 55 on endothelial, NK and T cells. HLA-G inhibits NK and T-cell mediated cell lysis and it can also induce CD4 positive T cell anergy and long-term immunotolerance. ${ }^{65}$ HLA-G is expressed by advanced ovarian cancer of aggressive histology ${ }^{66}$ and also correlates to a significantly worse prognosis in ovarian carcinoma. ${ }^{67}$

HLA-E may contribute to tumors escaping from immune surveillance by inhibiting NK cell-mediated lysis, since it is recognized by the CD94-NKG2 receptor on NK cells. ${ }^{68}$ Through highly specific peptide binding groove adapted to conserved leader sequence peptides on the HLA-E molecule, the NK cells are able to recognize cellular expression of 
other MHC class I molecules. HLA-E has been described as being over-expressed in several human cancers, particularly in colorectal cancer specimens that have decreased infiltration of NK cells as well as shorter disease-free survival..$^{69}$

\section{Class I-like genes: MICA/MICB}

The MHC class Ic-related genes A and B, MICA and MICB do not present peptides and are not associated with $\beta_{2}$-microglobulin. ${ }^{70}$ Instead MICA and MICB are polymorphic costimulating ligands for the receptor NKG2D on NK cells, CD8 ${ }^{+} \mathrm{T}$ cells and gamma/delta T cells and may activate both innate and adaptive immunity. ${ }^{71}$ MICA and MICB are present on the surface of many tumors and potentially may stimulate immune responses but can be downregulated by microRNA. ${ }^{72}$ Soluble MICA shed by tumor cells can bind to NKG2D receptor on NK and T cells without triggering downstream signals and induce degradation of the NKG2D receptor on the immune effector cells. ${ }^{73}$ Internalization of the complex also may result in expansion of immunosuppressive $\mathrm{NKG}_{2} \mathrm{D}^{+} \mathrm{CD} 4^{+} \mathrm{T}$ cells. There are potentially ligand-receptor interactions other than NKG2D that may also contribute to suppression of proliferating T cells. ${ }^{74}$

\section{The APM complex}

The APM complex, which mediates the interaction of tumor cells with HLA class I antigen restricted, tumor antigen (TA)-specific cytotoxic T lymphocytes, is generated through several steps with the participation of different molecules. Briefly, 9-11 amino acid-long peptides are derived from the cleavage of mostly, although not exclusively, endogenous cytosolic and nuclear proteins. The cleavage is executed by the constitutive and IFN- $\gamma$ inducible proteasome subunits as well as by other aminopeptidases. ${ }^{75}$ Peptides are then translocated via the heterodimeric transporter-associated with antigen processing (TAP) complex from the cytosol into the endoplasmic reticulum (ER), where they are loaded with the help of the chaperones calnexin, calreticulin, ERp57 and tapasin (tpn) onto $\beta 2$ microglobulin $\left(\beta_{2} \mu\right)$ HLA class I heavy chain (HC) complexes. The assembled trimeric HLA class I-peptide complex is released from the peptide-loading complex and transported via the trans Golgi apparatus to the cell surface for presentation to CD8 ${ }^{+}$cytotoxic T lymphocytes. ${ }^{76}$

\section{Defects in APM component expression}

The recently described positive outcome of a clinical trial with T-cell based immunotherapy in prostate cancer has stimulated interest in the characterization of the APM component expression in prostate cancer lesions, since this machinery plays a crucial role in the generation and expression of the trimeric HLA class I surface antigen complex on tumor cells. Defects in APM component expression have been described in several types of malignant diseases. ${ }^{77-80}$ They appear to have clinical relevance, since they can be associated with tumor progression, metastases formation as well as poor prognosis and decreased survival. To the best of our knowledge, only limited information is available about the MHC class I APM component expression in murine and human prostate cancer cells and its impact on immune responses. ${ }^{81-84}$ Murine primary and metastatic prostate cancer cell lines express MHC class I surface antigens despite the lack of detectable LMP2, LMP7, TAP1 and TAP2 transcription; IFN- $\gamma$ treatment induced these four APM components and enhanced MHC class I surface expression. ${ }^{82}$ The TAP defect in the murine metastatic prostate cancer cell line is caused by impaired initiation of TAP1 transcription. ${ }^{84}$ In particular, Sanda and coauthors $^{81}$ determined the constitutive and IFN$\gamma$-induced transcription of TAP2, $\beta_{2} \mu$ and HLA class I heavy chain, as well as the HLA class I surface antigen expression in five human prostate cancer cell lines. Lack of TAP2 expression in one metastatic human prostate cancer cell line resulted in loss of HLA class I antigen expression, which could be restored by IFN $-\gamma$. Two of the 5 analyzed cell lines did not express HLA class I antigens due to defects in their assembly or impaired histone acetylation, thereby escaping T-cell recognition. Similar defects were found in surgically removed prostate cancer lesions. Immunohistochemical staining of benign hyperplastic, primary and/or metastatic PC lesions with HLA class I- and/or $\beta_{2} \mu$-specific monoclonal antibodies (mAb) demonstrated a downregulation of these molecules in a high percentage of prostate lesions. ${ }^{85}$

In a study including 150 women with invasive ovarian cancers, loss of expression of different APM components could be correlated to decrease in survival. ${ }^{52}$ The results are similar to our study of Swedish HLA-A2-positive patients with advanced stage III-IV epithelial ovarian cancer of serous histology that consistently have poor prognosis despite treatment (Relationship between prognostic factor HLAA2 phenotype and presence of MHC and APM at the tumour tissue level in ovarian cancer patients, EA, LV, HH, KB, L Garberg, A Hoog et al., 2010, in preparation). This patient group who potentially require the greatest clinical monitoring, presents several abnormalities in pertinent immune-related factors in the tumor tissue, including the MHC class I HC and APM components. The impact of these defects, however, only assumes significance when considered cumulatively with negative prognostic value of tumor staging, histology and HLA-A2 genotype. There is evidence that increased copy number of HLA chromosomal region $6 \mathrm{p}$ is associated with a poor prognosis and more frequent in stage IIIc than in stage IIIa and IIIb ovarian tumors. Moreover, HLA chromosomal region $6 \mathrm{p}$ is associated with decreased expression of the corresponding HLA class II genes implying that there might be an unknown oncogene hidden in the $\mathrm{MHC}$ region which has an impact on disease outcome as well as surface expression. ${ }^{86,87}$

\section{The downregulation of HLA expression by oncogenes: the paradigm of HER-2/neu}

HER-2/neu is a $185-\mathrm{kDa}$ glycoprotein member of the epidermal growth factor receptor (EGFR) family of tyrosine kinases and considered one of the most attractive targets for immunotherapeutic interventions. ${ }^{88}$ This oncogene is selectively over-expressed in a broad variety of human tumors, including $25-40 \%$ of breast, head and neck cancer, ovarian, gastric, renal, oesophageal and colorectal carcinomas, and a small proportion of human melanomas. ${ }^{56,89-92}$ HER-2/ neu can be regarded as an indispensable tumor antigen, since tumours over-expressing HER-2/ neu are dependent on this oncogene for their survival and silencing consequently leads to growth arrest and/or apoptosis induction. ${ }^{88,93}$ This phenomenon is called "oncogene addiction", where a single oncogene can impair the growth and survival of cancer cells in spite of the fact that these cells contains a complexity of genetic and epigenetic abnormalities..$^{94}$ It is well known that HER-2/neu expression is associated with a reduced MHC class I surface antigen expression. ${ }^{88,95}$ HER-2/neu over-expression in murine and human tumor cells caused abnormalities of MHC class I surface expression due to impaired APM component expression, including LMPs, TAP and the chaperone tapasin, whereas the expression of MHC class I heavy chain as well as $\beta_{2} \mu$ were only marginally affected. This HER-2/neu-mediated deficient APM component expression could be reverted by IFN $-\gamma$ treatment suggesting a deregulation rather than structural alterations as underlying molecular mechanisms of their deficient expression. In order to determine the mode of regulation, the transcriptional activity of APM components was analyzed in HER-2/neu- and HER-2/neu+ cells using APM-specific promoter assays. All major APM components were transcriptionally down-regulated in HER-2/neu+ when compared to HER-2/neu cells, which was accompanied by a reduced binding of RNA polymerase II to the APM promoters. Sitedirected mutagenesis of $\mathrm{p} 300$, as well as the 
proximal and distal E2F1 transcription factor binding sites at position -146, and -229, resulted in a restoration of the tapasin promoter activity in HER-2/neu cells compared to that of wild-type promoter activity in untransformed HER-2/neu- fibroblasts. Moreover, siRNA-mediated silencing of transcription factor 1 (E2F1) is associated with an increased tapasin expression. These data suggest that a distinct expression pattern of transcription factors are involved in the suppression of APM-specific promoters and thus provides a novel route for intervention of HER-2/ neu ${ }^{+}$tumors (Bukur and Seliger, personal communication, 2010).

\section{Conclusions}

The MHC is remarkable in its profound impact on human diseases and its intriguing complexity with polymorphisms, duplications, insertions, deletions and yet strongly conserved blocks, known as ancestral haplotypes. Only recently has the role of HLA in cancer been established, considering that some haplotypes are connected to prognosis and survival in different types of cancer. The traditional role of MHC genes has been thought to be as signals for the cellular immune system and NK cells to recognize infected or neoplastic target cells. Recently, however, the non-coding sequences have gained more interest and the possibility of occult regulators of oncogenesis within this region is being examined. Attempts to link different HLA haplotypes to known oncogenes are also being carried out. One question that has only partly been addressed is the consequences of the high copy numbers of many MHC genes. The function of one gene may very well be counteracted on an epigenetic level if the same gene is duplicated or multiplied. Another issue is that haplotypes that are associated with poor prognosis in one malignancy are said to be protective in another.

One of the challenges in management of cancer patients is the limited knowledge of prognostic factors in patients with metastatic disease that predict their outcome following conventional therapy with surgery, radiation and chemotherapy. Our investigations validate the hypothesis that HLA typing of patients may provide an additional resource to predict with high probability the treatment outcome in patients with advanced ovarian, prostate cancers and malignant melanoma at diagnosis (Figure 1). Furthermore, the underlying molecular mechanisms that are utilized by tumor cells to deregulate HLA expression may provide novel opportunities for targeted therapy. In fact, our finding suggests that the HLAA2 genotype and ancestral haplotypes 62.1 and 8.1 are highly associated with poor prognosis and inadequate response to treatment. The

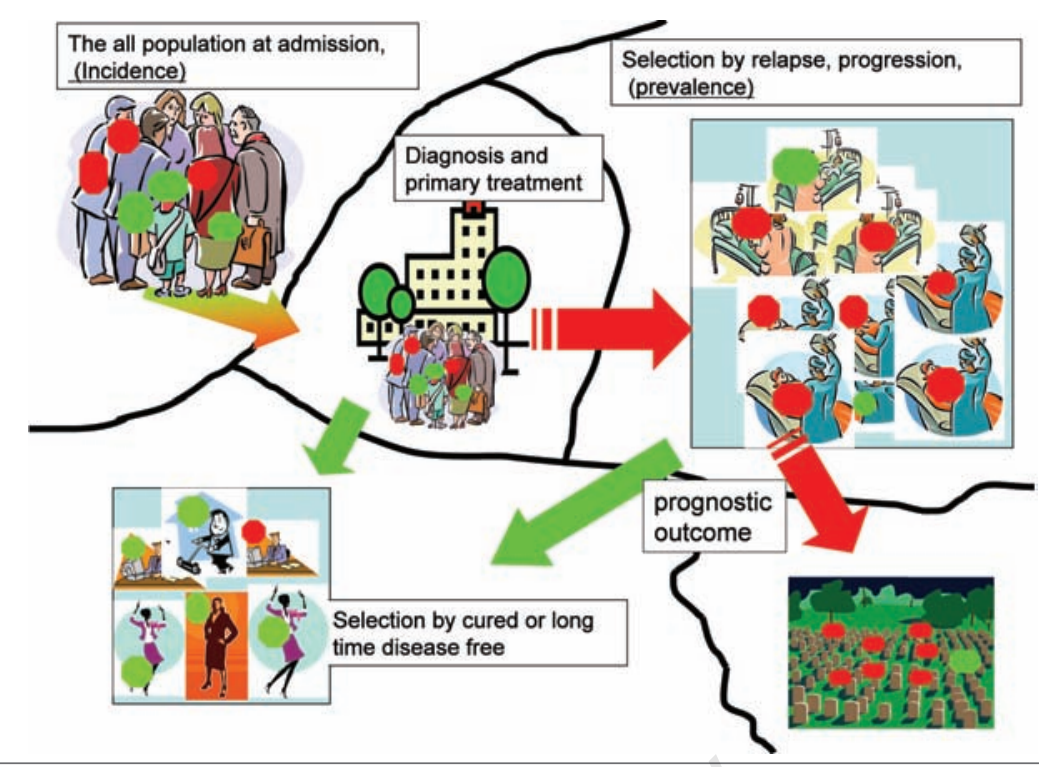

Figure 1. The population of healthy individuals (left upper corner) presents a normal distribution of HLA haplotypes (red spots= HLA A2 or AHH; green spots= others) when mapped against the theoretical risk of developing cancer (e.g. EOC, prostate cancer or malignant melanoma). No associations are noted. Following diagnosis (center) a significant number of those cases have long-term remissions or slow progressions (low left corner). The other group (red spots) have treatment-refractory and more aggressive disease. These patients demonstrate an increased frequency of HLA A2 and AHHs.

implications are that these patients can be identified in earlier stages before the development of metastatic disease and thereby provide a larger window of opportunity for intensive follow-up, early intervention and aggressive treatment. Patients with these unfavorable HLA types might benefit from more radical primary surgery, sentinel node biopsy if applicable, and more extensive adjuvant treatments. It is also critical to identify factors that can predict treatment response in patients with advanced disease. HLA typing might be a valuable tool to delineate patients who would benefit the greatest from aggressive regimes from those who have no additional benefit and increased adverse effects with more aggressive regimens. Additional therapeutic modalities that impede immune escape by tumors through downregulation of HLA expression may also increase the survival and prognosis of advanced cancer patients when utilized in combinatorial therapy approaches.

\section{References}

1. Townsend ARM, Gotch FM, Davey J. Cytotoxic T cells recognize fragments of the influenza nucleoprotein. Cell 1985;42:457.

2. Sioud M. Does our current understanding of immune tolerance, autoimmunity, and immunosuppressive mechanisms facilitate the design of efficient cancer vaccines? Scan J Immunol 2009;70:516-25.

3. Campoli M, Ferrone S. Tumor escape mech- anisms: potential role of soluble HLA antigens and NK cells activating ligands. Tissue Antigens 2008;72:321-34.

4. Browning M, Dunnion D. HLA and cancer: implications for cancer immunotherapy and vaccination. Eur J Immunogenet 1997;24:2 93-312.

5. Garrido F, Cabrera T, Aptsiauri N. "Hard" and "soft" lesions underlying the hla class I alterations in cancer cells: Implications for immunotherapy. Int J Cancer 2010;127:24956.

6. Seliger B. Molecular mechanisms of MHC class I abnormalities and APM components in human tumors. Cancer Immunol Immunother 2008;57:1719-26.

7. Blomhoff A, Olsson M, Johansson S, et al. Linkage disequilibrium and haplotype blocks in the MHC vary in an HLA haplotype specific manner assessed mainly by DRB1*03 and DRB1*04 haplotypes. Genes Immun 2006;7:130-40.

8. Reich DE, Cargill M, Bolk S, et al. Linkage disequilibrium in the human genome. Nature 2001;411:199-204.

9. Gabriel SB, Schaffner SF, Nguyen $\mathrm{H}$, et al. The structure of haplotype blocks in the human genome. Science 2002;296:2225-9.

10. Alper CA, Larsen CE, Dubey DP, et al. The haplotype structure of the human major histocompatibility complex. Hum Immun 2006;67:73.

11. Johnson GC, Esposito L, Barratt BJ, et al. Haplotype tagging for the identification of common disease genes. Nat Genet 2001; 29:233-7. 
12. consortium TMs. Complete sequence and gene map of a human major histocompatibility complex. The MHC sequencing consortium. Nature 1999;401:921-3.

13. Horton R, Wilming L, Rand V, et al. Gene map of the extended human MHC. Nat Rev Genet 2004;5:889-99.

14. Thorsby E. Invited anniversary review: HLA associated diseases. Hum Immunol 1997;53: 1-11.

15. Degli-Esposti MA, Leaver AL, Christiansen FT, et al. Ancestral haplotypes: conserved population MHC haplotypes. Human Immunology 1992;34:242.

16. Davidson EJ, Davidson JA, Sterling JC, et al. Association between human leukocyte antigen polymorphism and human papillomavirus 16-positive vulval intraepithelial neoplasia in British women. Cancer Res 2003;63:400-3.

17. Montoya L, Saiz I, Rey G, et al. Cervical carcinoma: human papillomavirus infection and HLA-associated risk factors in the Spanish population. Eur J Immunogenet 1998;25:329-37.

18. Cabrera T, Lopez-Nevot MA, Gaforio JJ, et al. Analysis of HLA expression in human tumor tissues. Cancer Immunol Immunother 2003; 52:1-9.

19. Chaudhuri S, Cariappa A, Tang M, et al. Genetic susceptibility to breast cancer: HLA DQB*03032 and HLA DRB1*11 may represent protective alleles. Proc Natl Acad Sci USA 2000;97:11451-4.

20. Garrity-Park MM, Loftus EV Jr, Sandborn WJ, et al. MHC Class II alleles in ulcerative colitis-associated colorectal cancer. Gut 2009;58:1226-33.

21. Lie AK, Skarsvag S, Haugen OA, et al. Association between the HLA DQB $1 * 0301$ gene and human papillomavirus infection in high-grade cervical intraepithelial neoplasia. Int J Gynecol Pathol 1999;18:206-10.

22. Niens M, Jarrett RF, Hepkema B, et al. HLA$A^{*} 02$ is associated with a reduced risk and HLA-A 01 with an increased risk of developing EBV+ Hodgkin lymphoma. Blood 2007; 110:3310-5.

23. Alexander FE, Jarrett RF, Cartwright RA, et al. Epstein-Barr Virus and HLA-DPB1-*0301 in young adult Hodgkin's disease: evidence for inherited susceptibility to Epstein-Barr Virus in cases that are EBV(+ve). Cancer Epidemiol Biomarkers Prev 2001;10:705-9.

24. Gamzatova Z, Villabona L, Dahlgren L, et al. Human leucocyte antigen (HLA) A2 as a negative clinical prognostic factor in patients with advanced ovarian cancer. Gynecol Oncol 2006;103:145-50.

25. Gamzatova Z, Villabona L, van der Zanden $\mathrm{H}$, et al. Analysis of HLA class I-II haplotype frequency and segregation in a cohort of patients with advanced stage ovarian cancer. Tissue Antigens 2007;70:205-13.
26. Helgadottir H, Andersson E, Villabona L, et al. The common Scandinavian human leucocyte antigen ancestral haplotype 62.1 as prognostic factor in patients with advanced malignant melanoma. Cancer Immunol Immunother 2009;58:1599-608.

27. Tisch M, Kyrberg $\mathrm{H}$, Weidauer $\mathrm{H}$, et al. Human leukocyte antigens and prognosis in patients with head and neck cancer: results of a prospective follow-up study. Laryngoscope 2002;112:651-7.

28. Hoon DS, Okamoto T, Wang HJ, et al. Is the survival of melanoma patients receiving polyvalent melanoma cell vaccine linked to the human leukocyte antigen phenotype of patients? J Clin Oncol 1998;16:1430-7.

29. Lee JE, Abdalla J, Porter GA, et al. Presence of the human leukocyte antigen class II gene DRB ${ }^{*} 1101$ predicts interferon gamma levels and disease recurrence in melanoma patients. Ann Surg Oncol 2002;9: 587-93.

30. Araria-Goumidi L, Lambert JC, Cottel D, et al. No association of the HLA-A2 allele with Alzheimer's disease. Neuroscience letters 2002;335:75-8.

31. Marincola FM, Venzon D, White D, et al. HLA association with response and toxicity in melanoma patients treated with interleukin 2-based immunotherapy. Cancer Res 1992; 52:6561-6.

32. Mitchell MS, Harel W, Groshen S. Association of HLA phenotype with response to active specific immunotherapy of melanoma. J Clin Oncol 1992;10:1158-64.

33. Sosman JA, Unger JM, Liu PY, et al. Adjuvant immunotherapy of resected, intermediate-thickness, node-Negative melanoma with an allogeneic tumor vaccine: impact of HLA class I antigen expression on outcome. J Clin Oncol 2002;20:206775 .

34. Bain C, Merrouche Y, Puisieux I, et al. Correlation between clinical response to interleukin 2 and HLA phenotypes in patients with metastatic renal cell carcinoma. Br J Cancer1997;75:283-6.

35. Franzke A, Buer J, Probst-Kepper M, et al. HLA phenotype and cytokine-induced tumor control in advanced renal cell cancer. Cancer Biother Radiopharm 2001;16:401-9.

36. Onishi $\mathrm{T}$, Ohishi $\mathrm{Y}$, Iizuka $\mathrm{N}$, et al. Phenotype frequency of human leukocyte antigens in Japanese patients with renal cell carcinoma who responded to interferonalpha treatment. Int J Urol 1996;3:435-40.

37. Cortes J, Fayad L, Kantarjian H, etal. Association of HLA phenotype and response to interferon-alpha in patients with chronic myelogenous leukemia. Leukemia 1998;12: 455-62.

38. De Petris L, Bergfeldt K, Hising C, et al. Correlation between HLA-A2 gene frequency, latitude, ovarian and prostate cancer mortality rates. Med Oncol 2004;21:49-52.

39. Nowak J, Kalinka-Warzocha E, Juszczynski $P$, et al. Association of human leukocyte antigen ancestral haplotype 8.1 with adverse outcome of non-Hodgkin's lymphoma. Genes Chromosomes Cancer 2007; 46:500-7.

40. Lewontin RC, Caspari EW. Developmental selection of mutations. Science 1960;132: 1688-92.

41. Deng S, Calin GA, Croce CM, et al. Mechanisms of microRNA deregulation in human cancer. Cell cycle 2008;7:2643-6.

42. Castelli EC, Moreau P, Oya E, et al. In silico analysis of microRNAS targeting the HLA-G 3 ' untranslated region alleles and haplotypes. Hum Immunol 2009;70:1020-5.

43. Liu Y, Zhao JJ, Wang CM, et al. Altered expression profiles of microRNAs in a stable hepatitis B virus-expressing cell line. Chin Med J (Engl) 2009;122:10-4.

44. Wang X, Gocek E, Liu CG, et al. Micro RNAs181 regulate the expression of p27Kip1 in human myeloid leukemia cells induced to differentiate by 1,25-dihydroxyvitamin D3. Cell cycle 2009;8:736-41.

45. Brown BD, Naldini L. Exploiting and antagonizing microRNA regulation for therapeutic and experimental applications. Nat Rev Genet 2009;10:578-85.

46. Chan JK, Loizzi V, Magistris A, et al. Differences in prognostic molecular markers between women over and under 45 years of age with advanced ovarian cancer. Clin Cancer Res 2004;10:8538-43.

47. Marincola FM, Wang E, Herlyn M, et al. Tumors as elusive targets of T-cell-based active immunotherapy. Trends Immunology 2003;24:335-42.

48. Waldhauer I, Steinle A. NK cells and cancer immunosurveillance. Oncogene 2008;27: 5932-43.

49. Norell H, Carlsten M, Ohlum T, et al. Frequent loss of HLA-A2 expression in metastasizing ovarian carcinomas associated with genomic haplotype loss and HLA-A2restricted HER-2/neu-specific immunity. Cancer Res 2006;66:6387-94.

50. Johnsen HE, Hutchings $M$, Taaning E, et al. Selective loss of progenitor subsets following clinical CD34+ cell enrichment by magnetic field, magnetic beads or chromatography separation. Bone Marrow Transplant 1999;24:1329-36.

51. Raffaghello L, Nozza P, Morandi F, et al. Expression and functional analysis of human leukocyte antigen class I antigenprocessing machinery in medulloblastoma. Cancer Res 2007;67:5471-8.

52. Han LY, Fletcher MS, Urbauer DL, et al. HLA class I antigen processing machinery component expression and intratumoral T-Cell infiltrate as independent prognostic markers in ovarian carcinoma. Clin Cancer Res 
2008;14:3372-9.

53. Kageshita T, Hirai S, Ono T, et al. Down-regulation of HLA class I antigen-processing molecules in malignant melanoma: association with disease progression. Am J Pathol 1999;154:745-54.

54. Ramnath N, Tan D, Li Q, et al. Is downregulation of MHC class I antigen expression in human non-small cell lung cancer associated with prolonged survival? Cancer Immunol Immunother 2006;55:891-9.

55. Vitale M, Pelusi G, Taroni B, et al. HLA class I antigen down-regulation in primary ovary carcinoma lesions: association with disease stage. Clin Cancer Res 2005;11:67-72.

56. Meissner M, Reichert TE, Kunkel M, et al. Defects in the human leukocyte antigen class I antigen processing machinery in head and neck squamous cell carcinoma: association with clinical outcome. Clin Cancer Res 2005;11:2552-60.

57. Mehta AM, Jordanova ES, van Wezel T, et al. Genetic variation of antigen processing machinery components and association with cervical carcinoma. Genes Chromosomes Cancer 2007;46:577-86.

58. Matsushita K, Takenouchi T, Shimada H, et al. Strong HLA-DR antigen expression on cancer cells relates to better prognosis of colorectal cancer patients: Possible involvement of c-myc suppression by interferongamma in situ. Cancer Sci 2006;97:57-63.

59. O'Callaghan CA, Bell JI. Structure and function of the human MHC class Ib molecules HLA-E, HLA-F and HLA-G. Immunol Rev 1998;163:129-38.

60. Le Bouteiller P, Blaschitz A. The functionality of HLA-G is emerging. Immunol Rev 1999;167:233-44.

61. Hviid TV. HLA-G in human reproduction: aspects of genetics, function and pregnancy complications. Hum Reprod Update 2006; 12:209-32.

62. Cai MY, Xu YF, Qiu SJ, et al. Human leukocyte antigen-G protein expression is an unfavorable prognostic predictor of hepatocellular carcinoma following curative resection. Clin Cancer Res 2009;15:4686-93.

63. Yie SM, Yang H, Ye SR, et al. Expression of human leucocyte antigen G (HLA-G) is associated with prognosis in non-small cell lung cancer. Lung Cancer 2007;58:267-74.

64. Tripathi P, Agrawal S. Non-classical HLA-G antigen and its role in the cancer progression. Cancer Invest 2006;24:178-86.

65. LeMaoult J, Krawice-Radanne I, Dausset J, et al. HLA-G1-expressing antigen-presenting cells induce immunosuppressive CD4+ T cells. Proc Natl Acad Sci USA. 2004;101: 7064-9.

66. Menier C, Prevot S, Carosella ED, et al. Human leukocyte antigen- $G$ is expressed in advanced-stage ovarian carcinoma of highgrade histology. Hum Immunol 2009;70:
1006-9.

67. Jung YW, Kim YT, Kim SW, et al. Correlation of human leukocyte antigen-G (HLA-G) expression and disease progression in epithelial ovarian cancer. Reprod Sci 2009;16:1103-11.

68. Hoare HL, Sullivan LC, Clements CS, et al. Subtle changes in peptide conformation profoundly affect recognition of the nonclassical MHC class I molecule HLA-E by the CD94-NKG2 natural killer cell receptors. J Mol Biol 2008;377:1297-303.

69. Levy EM, Bianchini M, Von Euw EM, et al. Human leukocyte antigen-E protein is overexpressed in primary human colorectal cancer. Int J Oncol 2008;32:633-41.

70. Stastny P. Introduction: MICA/MICB in innate immunity, adaptive immunity, autoimmunity, cancer, and in the immune response to transplants. Hum Immunol 2006;67:141-4.

71. Collins RW. Human MHC class I chain related (MIC) genes: their biological function and relevance to disease and transplantation. Eur J Immunogenet 2004;31:105-14.

72. Stern-Ginossar N, Gur C, Biton M, et al. Human microRNAs regulate stress-induced immune responses mediated by the receptor NKG2D. Nat Immunol 2008;9:1065-73.

73. Choy MK, Phipps ME. MICA polymorphism: biology and importance in immunity and disease. Trends Mol Med 2010;16:97-106.

74. Kriegeskorte AK, Gebhardt FE, Porcellini S, et al. NKG2D-independent suppression of T cell proliferation by $\mathrm{H} 60$ and MICA. Proc Natl Acad Sci USA 2005;102:11805-10.

75. Hammer GE, Kanaseki T, Shastri N. The final touches make perfect the peptide-MHC class I repertoire. Immunity 2007;26:397406.

76. Jensen PE. Recent advances in antigen processing and presentation. Nat Immunol 2007;8:1041-8.

77. Aptsiauri N, Cabrera T, Mendez R, et al. Role of altered expression of HLA class I molecules in cancer progression. Adv Exp Med Biol 2007;601:123-31.

78. Cabrera T, Lara E, Romero JM, et al. HLA class I expression in metastatic melanoma correlates with tumor development during autologous vaccination. Cancer Immunol Immunother 2007;56:709-17.

79. Marincola FM, Jaffee EM, Hicklin DJ, et al. Escape of human solid tumors from T-cell recognition: molecular mechanisms and functional significance. Adv Immunol 2000; 74:181-273.

80. Seliger B, Ritz U, Ferrone S. Molecular mechanisms of HLA class I antigen abnormalities following viral infection and transformation. Int J Cancer 2006;118:129-38.

81. Sanda MG, Restifo NP, Walsh JC, et al. Molecular characterization of defective antigen processing in human prostate cancer. J
Natl Cancer Inst 1995;87:280-5.

82. Lee HM, Timme TL, Thompson TC. Resistance to lysis by cytotoxic T cells: a dominant effect in metastatic mouse prostate cancer cells. Cancer Res 2000; 60:1927-33.

83. Sharpe JC, Abel PD, Gilbertson JA, et al. Modulated expression of human leucocyte antigen class I and class II determinants in hyperplastic and malignant human prostatic epithelium. Br J Urol 1994;74:609-16.

84. Setiadi AF, David MD, Chen SS, et al. Identification of mechanisms underlying transporter associated with antigen processing deficiency in metastatic murine carcinomas. Cancer Res. 2005;65:7485-92.

85. Kitamura $\mathrm{H}$, Torigoe $\mathrm{T}$, Asanuma $\mathrm{H}$, et al. Down-regulation of HLA class I antigens in prostate cancer tissues and up-regulation by histone deacetylase inhibition. J Urol 2007;178:692-6.

86. Partheen K, Levan K, Osterberg L, et al. Analysis of cytogenetic alterations in stage III serous ovarian adenocarcinoma reveals a heterogeneous group regarding survival, surgical outcome, and substage. Genes Chromosomes Cancer 2004;40:342-8.

87. Kubler K, Arndt PF, Wardelmann E, et al. Genetic alterations of HLA-class II in ovarian cancer. Int J Cancer 2008;123:1350-6.

88. Choudhury A, Kiessling R. Her-2/neu as a paradigm of a tumor-specific target for therapy. Breast Dis 2004;20:25-31.

89. Eliopoulos P, Mohammed MQ, Henry K, et al. Overexpression of HER-2 in thick melanoma. Melanoma Res 2002;12:139-45.

90. Al-Kuraya K, Novotny H, Bavi P, et al. HER2, TOP2A, CCND1, EGFR and C-MYC oncogene amplification in colorectal cancer. J Clin Pathol 2007;60:768-72.

91. Mimura K, Kono K, Hanawa M, et al. Frequencies of HER-2/neu expression and gene amplification in patients with oesophageal squamous cell carcinoma. Br J Cancer 2005;92:1253-60.

92. Seliger B, Rongcun Y, Atkins D, et al. HER2/neu is expressed in human renal cell carcinoma at heterogeneous levels independently of tumor grading and staging and can be recognized by HLA-A2.1-restricted cytotoxic T lymphocytes. Int J Cancer 2000;87: 349-59.

93. Choudhury A Kiessling R. Her-2/neu as a paradigm of a tumor-specific target for therapy. Breast Dis 2004;20:25-31.

94. Weinstein IB, Joe A. Oncogene addiction. Cancer Res 2008;68:3077-80; discussion 80.

95. Herrmann F, Lehr HA, Drexler I, et al. HER2/neu-mediated regulation of components of the MHC class I antigen-processing pathway. Cancer Res 2004;64:215-20. 